\title{
Philosophiques
}

\section{Nicolas de Béguelin et les fondements d'une philosophie de la nature}

\section{François Duchesneau}

Volume 42, numéro 1, printemps 2015

La philosophie à l'Académie de Berlin au XVIII ${ }^{\mathrm{e}}$ siècle

URI : https://id.erudit.org/iderudit/1032219ar

DOI : https://doi.org/10.7202/1032219ar

Aller au sommaire du numéro

\section{Éditeur(s)}

Société de philosophie du Québec

\section{ISSN}

0316-2923 (imprimé)

1492-1391 (numérique)

\section{Découvrir la revue}

\section{Citer cet article}

Duchesneau, F. (2015). Nicolas de Béguelin et les fondements d'une philosophie de la nature. Philosophiques, 42(1), 89-105. https://doi.org/10.7202/1032219ar

\section{Résumé de l'article}

Nicolas de Béguelin, philosophe et scientifique, membre de l'Académie de Berlin, entreprit de concilier des thèses contrastées sur les fondements de la philosophie de la nature, qui semblaient suggérer une antinomie irréductible entre les principes leibniziens-wolffiens et les principes newtoniens. Dans une série de mémoires consacrés à ce projet, il tente d'établir qu'une philosophie expérimentale reste incertaine de ses hypothèses, si elle ne les confronte aux réquisits qu'imposent certains des principes architectoniques dérivant du principe de la raison suffisante. Au coeur de l'argument figure une analyse visant à rattacher le concept de loi de la nature à la catégorie des vérités contingentes. Illustration en est fournie par la « déduction » des lois fondamentales de la mécanique : celles-ci ne sauraient être réduites à des propositions abstraites relevant de la seule nécessité géométrique. C'est en se fondant sur le réquisit de l'harmonie universelle que Béguelin propose des hypothèses sur l'intégration de forces motrices, intégration qui serait sous-jacente à l'inertie des corps, et sur l'espace comme ordre de coexistence des corps qui dépendrait de l'interrelation entre des éléments monadiques. 


\title{
Nicolas de Béguelin et les fondements d'une philosophie de la nature
}

\author{
FRANÇOIS DUCHESNEAU \\ Université de Montréal \\ francois.duchesneau@umontreal.ca
}

\begin{abstract}
RÉSUMÉ. - Nicolas de Béguelin, philosophe et scientifique, membre de l'Académie de Berlin, entreprit de concilier des thèses contrastées sur les fondements de la philosophie de la nature, qui semblaient suggérer une antinomie irréductible entre les principes leibniziens-wolffiens et les principes newtoniens. Dans une série de mémoires consacrés à ce projet, il tente d'établir qu'une philosophie expérimentale reste incertaine de ses hypothèses, si elle ne les confronte aux réquisits qu'imposent certains des principes architectoniques dérivant du principe de la raison suffisante. Au cœur de l'argument figure une analyse visant à rattacher le concept de loi de la nature à la catégorie des vérités contingentes. Illustration en est fournie par la «déduction» des lois fondamentales de la mécanique: celles-ci ne sauraient être réduites à des propositions abstraites relevant de la seule nécessité géométrique. C'est en se fondant sur le réquisit de l'harmonie universelle que Béguelin propose des hypothèses sur l'intégration de forces motrices, intégration qui serait sous-jacente à l'inertie des corps, et sur l'espace comme ordre de coexistence des corps qui dépendrait de l'interrelation entre des éléments monadiques.
\end{abstract}

\begin{abstract}
Nicolas de Béguelin, philosopher and scientist and a member of the Berlin Academy, undertook to conciliate such conflicting views concerning the foundations of natural philosophy as seemed to suggest an irreducible antinomy between the Leibnizian-Wolffian and the Newtonian principles. In a series of memoirs, he argued that experimental philosophy would remain unsettled about the validity of its hypotheses as long as it failed to check them against requisites arising from architectonic principles and their source in sufficient reason. At the heart of his argument, one finds an analysis of the relationship that holds between laws of nature and contingent truths. Case studies were provided relative to the "deduction" of the fundamental laws of mechanics: these should be in no way equated with abstract statements featuring geometrical necessity. Relying on the requisites of universal harmony, Beguelin framed up hypotheses concerning the integration of motive forces underpinning inertia, as well as concerning space as an order of coexistent bodies that would depend on the interrelation between monadic elements.
\end{abstract}

Nicolas de Béguelin (I7I4-I789) compte parmi les philosophes de l'Académie de Berlin, dont il devint membre en $\mathrm{I} 747^{1}$. Suisse d'origine, formé aux sciences dans le cercle des Bernoulli à Bâle, il fut un brillant polymathe

1. Voir Samuel Formey, "Éloge de M. de Béguelin", Mémoires de l'Académie royale des sciences et des belles-lettres, I788-I789, Berlin, Decker, I793, p. 39-50; Paul Dumont, Nicolas de Béguelin (I7I4-I789). Fragment de l'histoire des idées philosophiques en Allemagne dans la seconde moitié $d u$ XVIII ${ }^{e}$ siècle, Neuchâtel, Impr. de Attinger frères, I907.

PHILOSOPHIQUES 42/1 — Printemps 2015, p. 89-105 
auquel on a parfois reconnu d'intéressantes contributions en mathématiques ainsi qu'en physique, et notamment en optique, en ce qui concerne la théorie de la lumière qu'il entreprend de réviser par-delà les modèles initialement fournis par Newton, et par Huygens et Euler. En philosophie, le trait marquant de ses travaux, pour l'essentiel publiés parmi les mémoires de l'Académie de Berlin, tient à sa volonté de concilier la philosophie héritée de Leibniz avec les positions apparemment antithétiques soutenues par les successeurs et les épigones de Locke. D'où la critique facile consistant à dénoncer, chez lui et dans son œuvre, un syncrétisme consistant à harmoniser les points de vue par juxtaposition, plutôt qu'à opérer une véritable synthèse des perspectives. On peut aussi lui reconnaître le mérite de n'avoir pas confondu les thèses leibniziennes et wolffiennes, lorsqu'elles divergeaient. Mais il y a sans doute plus à retenir que ces seules caractéristiques d'une démarche originale visant à fournir des principes métaphysiques à une science de la nature qui apparaissait indûment restreinte aux inductions de la philosophie expérimentale, ainsi qu'aux hypothèses explicatives de portée limitée qui correspondaient à cette méthodologie. Aussi me semble-t-il utile de déterminer le rapport qu'il convient d'établir entre les recherches théoriques auxquelles Béguelin s'est livré en philosophie de la nature, et l'application qu'il a faite d'une méthodologie de type leibnizien à l'analyse et à l'explication de phénomènes physiques. Pour en traiter, je m'intéresserai aux principaux mémoires de Béguelin relatifs aux fondements d'une philosophie de la nature, et je tenterai de montrer comment les postulats métaphysiques et l'analyse épistémologique s'y conjuguent.

\section{Quel fondement pour les lois de la mécanique?}

De 1755 à I768, Béguelin présente à l'Académie de Berlin une série de cinq mémoires consacrés "aux premiers principes de la métaphysique ${ }^{2}$ » Le dernier de ces mémoires s'intitule De l'Usage du principe de la raison suffisante dans les loix générales de la mécanique ${ }^{3}$. Béguelin y part du statut des lois du mouvement selon d'Alembert: le mathématicien français leur attribuait le caractère de vérités nécessaires suivant un concept de nécessité qui les assimilait à des principes mathématiques, inférés de la notion abstraite de réalité matérielle et exprimant symboliquement les propriétés déterminantes de celle-ci ${ }^{4}$. Partant de ces principes, les implications du concept se déploieraient

2. Les quatre premiers mémoires s'intitulaient "Mémoire sur les premiers principes de la métaphysique ", Histoire, I757, p. 405-423; "Second mémoire sur les principes métaphysiques ", ibid., p. 424-447; "Troisième mémoire. De l'Usage légitime du principe de la raison suffisante ", Histoire, I768, p. 325-340; "Sur l'usage du principe de la raison suffisante dans le calcul des probabilités ", Histoire, I769, p. 382-4I 2.

3. Nicolas Béguelin, «De l'Usage du principe de la raison suffisante dans les loix générales de la mécanique ", Histoire, I770, p. 367-383.

4. Voir Jean Le Rond D'Alembert, Essai sur les éléments de philosophie (1759), Hildesheim, Olms, I965, $\$$ XVI, Mécanique, p. 367-40I. 
par voie de conséquences nécessaires. Selon Béguelin, le recadrage épistémologique de ces lois doit se faire par référence aux notions leibniziennes de vérité nécessaire et de vérité contingente, et suivant la juridiction distincte des deux domaines rationnels, respectivement par le principe de contradiction et par celui de la raison suffisante. Or les lois de l'équilibre et du mouvement relèvent de ce dernier domaine en tant que vérités contingentes. Se référant à l'article 349 des Essais de théodicée (I7IO) de Leibniz ${ }^{5}$, Béguelin soutient:

Le caractère de contingence de ces loix est d'avoir ph, absolument parlant, même en admettant l'existence de la matiere et du mouvement, être autres qu'elles ne sont, $\&$ de n'avoir pas $d \hat{u}$ être; de découler non d'une nécessité invincible, non d'une volonté arbitraire du Créateur, mais des Loix supremes de la convenance, de cette raison éternelle qui dirige les décrets d'un Etre infiniment sage ${ }^{6}$.

Pour justifier ce rapport des principes mécaniques à une raison supérieure de convenance selon Leibniz, il n'hésite pas à y assimiler l'usage téléologique que Maupertuis faisait du principe de moindre action dans son Essai de cosmologie (1750) ${ }^{7}$.

Mais il importe surtout à Béguelin de dévoiler les apories qui résulteraient d'une réduction des principes de la mécanique aux implications nécessaires de concepts abstraits ayant un statut analogue à celui des notions de nombre et d'étendue. Tout se passerait donc comme si les trois lois fondamentales et indépendantes auxquelles se réduisent les principes de la mécanique selon d'Alembert - loi de l'inertie, loi de la composition des forces et loi de l'équilibre - n'étaient que des suites nécessaires du concept abstrait d'impénétrabilité des corps. Dans le même temps toutefois, ce concept ne saurait être considéré comme énonçant une relation d'identité purement arbitraire sans correspondance avec la réalité des choses, comme Béguelin le trouve suggéré dans un mémoire de Daviet de Foncenex ${ }^{8}$, car cela reviendrait à diluer totalement la notion de lois qui seraient conformes à l'ordre naturel et aptes à le régir. La stratégie déployée par Béguelin pour résoudre l'aporie consiste essentiellement à montrer, d'une part, que le principe de l'inertie ne saurait découler par implication nécessaire de la notion de matière impénétrable, et d'autre part, que toute modalité d'application du

5. G. W. Leibniz, Die philosphischen Schriften, hrsg. von C. I. Gerhardt, Hildesheim, Olms, I965, VI, p. 32I-322.

6. Béguelin, De l'Usage..., p. 369.

7. Ici Béguelin s'appuie manifestement sur le mémoire de Pierre Louis Moreau de Maupertuis, "Examen philosophique de la preuve de l'existence de Dieu employée dans l'Essai de cosmologie ", Histoire, I758, p. 389-424.

8. François Chevalier Daviet de Foncenex, "Sur les principes fondamentaux de la méchanique ", in Mélanges de philosophie et de mathématique de la Société royale de Turin, pour les années I760-I76I, Turin, De l'Imprimerie royale, s.d., $2^{\mathrm{e}}$ partie, p. 299-322. 
principe à des effets de l'ordre naturel requiert que l'on recoure à une raison de la relation concernée, par-delà le type de permanence d'état de repos ou de mouvement qu'implique le concept d'inertie.

Le système d'arguments ici développé rejoint une considération architectonique intéressante: Béguelin distingue une question de fait et une question de rapport à l'ordre harmonique et systémique des concepts dont on se sert pour figurer la compatibilité des diverses caractéristiques phénoménales et les subordonner à une loi d'ensemble. Rejetant la conception purement nominale de la loi, Béguelin en rattache l'analyse soit à une simple validation empirique, soit à une architecture de raisons assurant la liaison entre la relation d'inertie et les propriétés associées à la définition de la nature corporelle: cette relation ne peut être fondée sur une simple implication logique, mais au contraire sur un système de raisons suffisantes?

De multiples arguments sont ici évoqués. Il me suffira de souligner ce qui y prédomine. L'attribution au principe d'inertie d'une nécessité géométrique va de pair avec la supposition d'une matière essentiellement passive dont les états de repos et de mouvement seraient des déterminations ou «relations extrinsèques et accidentelles ${ }^{10}$ ». Se pose alors la question de la continuité d'exercice de la force (supposée externe) dans la continuation de l'état de mouvement d'un corps (supposé inerte). Il semble, dans les conditions présumées de l'expérience, que l'on ne puisse se dispenser d'admettre une cause assurant le changement perpétuel et uniforme d'état, cause que semble requérir l'effet inertiel en tant que raison déterminante. Par ailleurs, il nous est loisible de concevoir qu'une infinité de relations autres que celle de l'inertie pourraient être rattachées sans contradiction au concept de nature corporelle duquel on présumerait à tort que la relation d'inertie découle nécessairement. Les systèmes ainsi hypostasiés ne souffriraient d'aucune incompatibilité logique par rapport à ce dernier concept. Ils pourraient seulement représenter un ordre déficient de raisons suffisantes par rapport à l'ordre physique réalisé ${ }^{11}$. Il serait certes contradictoire que le même corps, en même temps et sous le même rapport, fût en repos et en mouvement, mais la continuation d'état et le changement auquel cet état est susceptible d'être soumis par suite de l'interaction des corps supposent une raison d'intégration des mouvements en un ensemble cohérent, réglé, déterminé et constant: cette raison d'intégration se déploierait à l'échelle du système que forment les corps en vertu de l'ordre de convenance qui les détermine respectivement les uns par rapport aux autres.

Tout changement d'état dans la nature a besoin d'une raison suffisante: telle est la loi d'inertie: dès-là tous les corps qui agissent les uns sur les autres déploient \& éprouvent une force proportionnée au changement qui s'opere

9. Voir Béguelin, De l’Usage..., p. 373.

10. Ibid., p. 374 .

11. Voir ibid., p. 375. 
sur eux tous. Ainsi le changement total qui arrive dans la nature suppose une force déterminée par le besoin d'une raison suffisante; force par conséquent la plus petite qu'il soit possible pour produire ce nouvel état ${ }^{12}$.

Béguelin opère le même type d'analyse au sujet des deux autres lois générales telles que d'Alembert les avait formulées: celle de la composition et de la décomposition des forces, et celle de l'équilibre. En ce qui a trait à la loi de la composition, il fait essentiellement valoir que la détermination de la direction pour le corps en mouvement, tout comme celle de la quantité de mouvement qu'il déploie, ne saurait découler géométriquement de la considération du corps, non plus que du mouvement, mais suppose un principe de choix entre systèmes possibles, car le lien de la loi aux concepts abstraits impliqués relève d'une raison supérieure de convenance dont la découverte irait de pair avec l'expérience des faits.

En ce qui concerne le principe de l'équilibre, il semblerait à première vue que ce soit une relation d'égalité des forces qui en assure l'articulation interne, or, l'égalité renvoyant à l'identité, une apparence de vérité nécessaire paraîtrait s'y rattacher. Mais, de fait, cette loi implique une seconde relation suivant laquelle des forces égales produiraient des effets égaux, si du moins elles étaient appliquées à des distances égales du point d'appui. Or ce ne serait a priori pas une contradiction que des forces diverses produisissent le même effet, ni que, pour des distances inégales d'application des forces, le même rapport fût conservé. Par suite, la loi doit renvoyer à une raison spécifique de convenance. En outre, l'égalité des mouvements et des vitesses réalisés par les corps en déplacement se trouve rapportée à un état d'équilibre dans lequel mouvements et forces n'apparaissent qu'en puissance et comme sous-tendant de façon particulière l'état de repos. Toutes ces considérations nous renvoient en fait à une liaison de raisons suffisantes impliquant une continuité d'ordre dans les rapports divers dont la loi vise à rendre compte par-delà le cas-limite de l'équilibre. Citant l'article 345 des Essais de théodicée, Béguelin rappelle que ces lois "ne naissent pas entièrement du principe de la nécessité, mais [...] naissent du principe de la perfection et de l'ordre ${ }^{13}$ ». Il trouve en outre confirmation de sa thèse dans le fait que les lois du mouvement ainsi déterminées se révèlent en accord par leurs effets avec le principe de la moindre action selon Maupertuis, qu'il considère, par une sorte de réappropriation leibnizienne, semble-t-il, comme un principe non seulement téléologique en un sens épistémique, mais aussi architectonique et signifiant l'ordre causal de l'univers.

S’il repense ainsi les principes fondamentaux de la mécanique en les justifiant comme des vérités contingentes requérant une garantie métaphysique,

12. Ibid., p. 377 .

13. Ibid., p. 382-383, voir Leibniz, Die philosophischen Schriften, VI, p. 3 I9. 
Béguelin va au-delà dans sa tentative d'application du principe de raison suffisante en physique.

\section{Quel fondement pour la force et la composition des corps?}

Soutenant méthodologiquement la légitimité de recourir aux principes architectoniques pour rendre compte des lois de la nature, Béguelin entendait fournir une base métaphysique à une philosophie de la nature qui était largement dominée en son temps par les lois et les concepts empiriquement fondés de la physique newtonienne. D’où le projet des trois mémoires voués à cet objectif, qui paraissent dans l'Histoire de l'Académie royale des sciences et des belles-lettres pour les années I766 et I769. Dans l'Essai d'une conciliation de la métaphysique de Leibnitz avec la physique de Newton ${ }^{14}$, il justifie cette démarche en évoquant l'utilité de former des hypothèses dont le potentiel d'explication serait à la fois sanctionné par l'emploi de la raison et par le recours à l'expérience, ce qui nous empêcherait de craindre d'aboutir par cette démarche à de vaines spéculations. L'analyse qu'il mène dans ce premier mémoire porte sur deux sujets sur lesquels s'affrontent les programmes newtonien et leibnizien : la composition des corps et leur pesanteur. Béguelin inventorie les divers concepts en jeu dans cet affrontement théorique. On a affaire, d'une part, aux concepts apparemment incompatibles de monades, d'atomes ou d'éléments infiniment divisibles en ce qui concerne les corps, aux concepts d'attraction (propriété interne), d'impulsion ou de pression (principe causal externe), d'autre part, en ce qui concerne la pesanteur. Dans ce dernier cas, reconnaissons que l'hypothèse newtonienne de l'attraction est conceptuellement problématique, mais parfaitement conforme par ses implications aux phénomènes dont il s'agit de rendre compte. À l'inverse, en quelque sorte, si les explications mécaniques de la gravitation ne renvoient qu'à des concepts pleinement intelligibles, les modèles tourbillonnaires que l'on a proposés à la suite de Descartes et de Leibniz se révèlent difficilement conciliables avec la totalité des phénomènes que l'on tente d'y rattacher, notamment en raison des propriétés ad hoc dont on doit, pour y parvenir, doter la matière subtile, impondérable, extrêmement pénétrante et sujette à des déterminations motrices inexplicables. Et de fait, Leibniz et Newton ont développé des vues antagonistes en raison des inférences qu'ils ont tirées des concepts qu'ils ont hypostasiés.

Peut-on envisager une forme de conciliation entre ces développements systématiques qui semblent s’opposer ? Béguelin, entreprenant cette conciliation, suppose que les divergences entre les deux savants étaient essentiellement méthodologiques. Il faut entendre par là que leurs démarches de recherche se profilaient respectivement à des niveaux distincts, l'un physique,

14. Nicolas Béguelin, «Essai d'une conciliation de la métaphysique de Leibnitz avec la physique de Newton, d'où résulte l'explication des phénomenes les plus généraux et les plus intéressans de la nature ", Histoire, I768, p. 365-380. 
l'autre métaphysique, et que les hypothèses en conflit étaient construites différemment, selon qu'elles devaient être soumises au contrôle des seules représentations empiriques ou subordonnées à des principes architectoniques. Certes, le défi est considérable, s'agissant de concilier le système de l'attraction et celui des monades. Mais il est déjà apparu que les estimes respectives de la force motrice dans les deux systèmes pouvaient être acceptées suivant le point de vue adopté sur les phénomènes à expliquer, qu'il fût leibnizien ou newtonien. Et Béguelin se dit persuadé que l'on pourrait aussi résoudre de cette façon les apories relatives aux concepts cosmologiques tels que ceux d'espace, de temps et de plein ou de vide, par une forme de corrélation des points de vue au sein d'une sorte de synthèse supérieure. Et c'est ce qu'il entend tenter en ce qui concerne l'antinomie des concepts représentant les propriétés et les éléments constitutifs des réalités corporelles.

La conciliation visée s'appuie sur la reprise et la révision du concept de monade. Celui-ci est d'une part rattaché à la grande chaîne des êtres en vertu du principe de continuité. Par-delà les révisions de Wolff qui ont mené à un concept d' «élément» dénué de capacité représentative, la perception est identifiée comme propriété essentielle de toute monade et interprétée dans le sens d' " un sentiment [qui peut être] très obscur de soi-même et du reste de l'univers ${ }^{15}$ ". Quant à l'appétition monadique, d'une tendance à percevoir et à changer de perception qu'elle était chez Leibniz elle devient une tendance à l'union avec les autres monades suivant des rapports de plus ou moins grande affinité. L'argument est d'abord développé sur la base d'expériences anthropologiques présumées: tant chez les hommes que chez les animaux, les individus se constitueraient en sociétés par suite d'un "penchant social». Le principe de continuité implique des effets et des causes similaires dans l'interaction des corps, là où l'on assiste à des combinaisons chimiques d'éléments de même nature, ainsi qu'à des processus de cristallisation que l'on ne saurait expliquer que par des coalescences de particules résultant de relations diverses d'affinité. Le modèle présume une caractéristique additionnelle des éléments de la nature. Plus les monades sont de degré supérieur, plus elles discriminent entre les rapports d'union à actualiser en privilégiant le rapport aux êtres de même nature. En descendant l'échelle des êtres, l'on assisterait à une tendance à l'association de particules qui serait plus générale et moins spécifique ${ }^{16}$.

Le modèle hypothétique appliqué aux éléments inférieurs du système de la nature, ceux qui se rattachent à la matière brute, se trouve représenté par la supposition de deux masses de matière, telles que la Terre et la Lune. Dans la composition de ces masses entrent une infinité d'éléments qui seraient dans des rapports de proximité spatiale en raison du sentiment obscur qui les déterminerait à s'associer aux autres éléments pour former des

15. Ibid., p. 373 .

16. Voir ibid., p. 375. 
ensembles corporels infiniment diversifiés. La détermination des éléments les uns vers les autres s'exprimerait par des tendances susceptibles de sommation pour donner les effets moteurs attribuables à la pesanteur particulière, celle que mesurent des rapports conformes à la loi de Galilée sur la chute des corps et s'appliquant à proximité de la surface du globe. Et le même système d'analogies permettrait mutatis mutandis de rendre compte des effets de la gravitation pour des masses corporelles interagissant à des distances variables l'une de l'autre.

Ce que Béguelin vise à montrer, c'est que les relations mécaniques liant les corps en interaction correspondent, dans un registre plus fondamental, aux effets de l'entre-expression des monades, pour autant que l'on envisage des systèmes où les rapports élémentaires monadiques engendreraient des phénomènes plus globaux dont la mesure serait par ailleurs conforme aux lois empiriques de la physique moderne. Ces lois se justifieraient en vérité, sur un plan plus métaphysique, par les implications d'un modèle de monadologie physique ou physiologique: celui-ci fournirait la raison suffisante d'un système d'affinités différentielles entre les propriétés de corps déployant leurs effets conformément à un principe d'harmonie universelle.

Cette façon de résoudre les apories de la philosophie naturelle ne peut fonctionner que si l'on présume que la validité des explications scientifiques repose sur des critères de simple conformité aux mesures opérées sur les phénomènes, et que la validité des hypothèses métaphysiques repose sur le développement de systèmes de concepts reliés par des jeux d'analogie architectonique. Il s'agirait, dans ce deuxième cas, d'une sorte de construction spéculative qui rejoindrait, par ses implications, les données thématisées par les lois de la physique expérimentale - ce que traduit assez la déclaration suivante:

Newton est conduit par la route des Phénomenes aux loix d'une attraction inconcevable à la raison, \& qui montrée de ce côté-là révolte la Philosophie de Leibnitz; celui-ci découvre par la voie du raisonnement des Etres simples, qui paroissent autant de chimeres à l'esprit géométrique du Philosophe anglois. Il n'y a cependant que ces monades, qui puissent rendre intelligible l'attraction de la matiere, \& c'est cette attraction vérifiée par la course de tous les corps célestes, qui fournit l'unique preuve palpable de l'existence des monades. On pourroit donc dire que Leibnitz, en donnant la solution du probleme sur la nature des corps, a trouvé d'avance la cause d'une attraction qu'il ne connoissoit point encore, \& que Newton, en découvrant cette gravitation \& ses loix, a donné la démonstration physique de l'existence des Etres simples qu'il n'admettoit pas ${ }^{17}$.

À certains égards, l'on se rapproche davantage d'une problématique relative aux seuls fondements de la physique si l'on prend en compte la tentative, dont le mémoire lu le I 6 mars I 769 fait état, visant à résoudre l'aporie 
créée par l'attribution aux corps de deux propriétés essentielles: l'inertie, d'une part, et la propension constante au changement d'état, d'autre part ${ }^{18}$. Il s'agit alors de justifier la position leibnizienne à l'encontre des nombreuses critiques qui tendaient à y déceler une contradiction flagrante, critiques parmi lesquelles figurent celles qu'Euler adressait à la cosmologie wolffienne ${ }^{19}$. Tout compte fait, Béguelin reproche à Euler d'avoir limité son analyse à une mécanique conforme aux seules normes de l'analyse géométrique, sans s'élever aux raisons suffisantes susceptibles, par l'évocation de leur source métaphysique, de rendre compte des lois ainsi formulées:

La plus grande force dans l'art de résoudre des équations compliquées ne fera jamais découvrir une vérité bien intéressante, si la Métaphysique n’a pas déterminé d'avance par les discussions les plus délicates toutes les conditions du probleme $^{20}$.

Le double objectif de Béguelin est donc à la fois d'établir la cohérence des deux concepts dans la dynamique leibnizienne et de déterminer comment les deux propriétés peuvent être liées dans l'économie d'une physique conforme à l'expérience.

Béguelin évoque indirectement les analyses du Specimen dynamicum ( 695$)^{21}$ de Leibniz en rappelant d'une part que tous les éléments simples selon le lexique wolffien - fondent ce qu'il y a de substantiel dans les corps et possèdent une force active qui leur est propre et qui varie constamment les états de ces éléments, sans qu'il y ait d'interaction entre eux, si ce n'est par correspondance harmonique. En ce qui concerne «l'effet sensible ou physique $^{22}$ » de leur action, il se révèle dans l'ordre des phénomènes comme s'il y avait action et réaction des corps constituant le monde, les uns à l'égard des autres, mais avec cette caractéristique particulière que les corps phénoménaux agiraient au dehors en vertu de la force physique dont ils seraient doués: comprenons qu'il s'agit de la «force dérivative» au sens leibnizien.

Sur cette base, le modèle que propose Béguelin pour concilier force physique autonome et inertie est ingénieux. Un corps constitue un tout dans l'ordre phénoménal dans la mesure où il est composé d'une combinaison d'éléments formant un système suffisamment intégré pour qu'il possède un centre de gravité commun. Le mouvement déterminé ou le repos de ce centre de gravité représente l'état inertiel du système que compose tout

18. Nicolas Béguelin, "Sur deux propriétés des corps, qui semblent incompatibles; l'inertie et la tendance au changement d'état ", Histoire, I77 I, p. 335-343.

19. Voir en particulier Leonhard Euler, "Sur la nature des moindres parties de la matiere", Histoire, I746, p. 28-32; "Essai sur la formation des corps en général ", Histoire, I750, p. 3-I 5; «Reflexions sur l'espace et le tems », ibid., p. 324-333.

20. Béguelin, Sur deux propriétés, p. 336.

21. G. W. Leibniz, Mathematische Schriften, hrsg. von C. I. Gerhardt, Hildesheim, Olms, I97I, VI, p. 234-246.

22. Béguelin, Sur deux propriétés, p. 337. 
corps, compris comme la résultante d'une combinaison intégrée d'éléments. Or cette combinaison intégrée provient précisément de la composition des forces actives dont sont dotées les parties constitutives du système. Et ces parties constitutives peuvent à leur tour être vues comme des systèmes d'éléments renvoyant à des systèmes d'éléments qui en constitueraient les composantes en quelque sorte plus élémentaires. Et ainsi de suite jusqu'à l'ordre infinitésimal qui ne serait jamais empiriquement atteignable. L'inertie apparaît dans ce modèle comme un corrélat de l'interaction des corps, interaction découlant elle-même de l'unité véritable des corps, substantiellement fondée sur la possession intrinsèque d'une force active de degré donné.

Dans tous les cas l'inertie du système entier résultera des forces actives de toutes les parties qui le constituent. Par la même raison l'inertie du plus petit atome de matiere peut résulter du concours ou du conflit apparent des forces élémentaires dont cet atome représente confusément à nos sens la combinaison; ou, ce qui revient au même, cet atome matériel n'étant que l'expression obscure \& sensible d'un système d'éléments simples, l'inertie de cet atome est analogue à celle du centre de gravité d'un nombre quelconque de grands corps qui agissent entr'eux : \& par conséquent, dans les deux cas, cette inertie tire sa source des forces dont chaque puissance qui concourt à former le système est animée ${ }^{23}$.

Ce modèle représente un intérêt additionnel du point de vue du fondement strict de la théorie physique: il semble permettre de trancher la question de la compatibilité de l'inertie et de la force active intrinsèque sans aborder la question ontologique fondamentale de savoir si la décomposition des corps peut s'arrêter à des atomes matériels ou si elle doit impliquer des éléments simples de type monadique, corrélatifs d'une décomposition à l'infini.

Toutefois, la seconde question relative à la coexistence effective des deux propriétés dans les corps ne peut être réglée sur la base du seul recours à l'expérience et requiert que l'on aborde la question d'un point de vue proprement métaphysique. Dans la perspective restreinte d'une physique expérimentale, on peut certes tenir indifféremment l'inertie pour une propriété essentielle au corps et la force active pour une propriété adventice découlant de quelque action mécanique externe qui s'exercerait sur le corps, ou l'inverse. Il peut en effet suffire au physicien de constater l'effet de telles propriétés et d'en noter les lois d'application, telles qu'une mise en forme mathématique des données d'expérience permet de les formuler. La visée de Béguelin se situe au-delà de ces limites épistémologiques. Si les hypothèses de type ontologique se valent pour le physicien qui ne s'y intéresse que pour les mettre en quelque sorte entre parenthèses, il n'en est pas de même pour le philosophe qui cherche à établir un système de raisons suffisantes aussi complet que possible afin d'assurer l'intégration des diverses lois empiriques 
à un même cadre spéculatif. Dans ce contexte, l'hypothèse leibnizienne suivant laquelle la force active est essentielle aux éléments du corps, et l'inertie une propriété découlant de la combinaison des forces élémentaires, offre des avantages théoriques notables. Et c'est là un motif de discrimination en sa faveur.

Ainsi le modèle rend-il compte de l'impénétrabilité des corps suivant une gradation conforme aux forces actives correspondantes, ce que l'hypothèse adverse transformerait en une sorte de postulat arbitraire. La difficulté principale à surmonter tient néanmoins à une apparente insuffisance du modèle à impliquer ontologiquement l'inertie dans un système où il semblerait que la moindre puissance externe pût et dût modifier l'état d'équilibre résultant de l'action des éléments combinés. Sans le suggérer, Béguelin retrouve ici l'aporie de la simple sommation algébrique des conatus dans la première mécanique abstraite de Leibniz ${ }^{24}$. Pour pallier cette déficience présumée, il faut réintroduire ici une propriété positive d'inertie dans l'essence des corps, propriété dont l'existence relève d'une vérité contingente, et non plus d'un simple réquisit découlant d'une abstraction mathématique. Somme toute, Béguelin propose de considérer qu'il convient à l'ordre physique que l'inertie y figure comme une condition contingente de l'interaction dynamique des corps:

Mais, pour qu'il regne un ordre constant dans la nature, pour qu'il y ait une vérité déterminée dans la suite des évenemens, la Raison supreme vouloit qu'il y eût une proportion entre les causes $\&$ les effets, que tous les degrés de mouvement ne résultassent pas d'une impulsion quelconque; qu'un atôme de poussiere tombant sur un bras de la balance à un pouce de distance du point d'appui, ne donnât pas à deux poids de cent livres chacun une secousse égale à celle qu'ils recevroient de la chûte d'une bombe qui tomberoit sur ce même bras, à deux pieds de son appuii ${ }^{25}$.

La solution va consister à postuler que toute action externe s'exerçant sur le corps conçu comme système, pour le changer, se voit opposer une puissance de résistance inhérente aux éléments du système. Le déploiement de force requis pour sortir le système de son état d'équilibre suscite la rénitence du système. Béguelin suppose cette rénitence infiniment petite dans les moindres éléments de la nature supposés intrinsèquement actifs, mais ces éléments sont susceptibles de sommations à divers degrés dans les combinaisons d'éléments d'où émergent les corps; et, dans ces combinaisons, la masse doit être prise en compte lors du calcul des effets inertiels dus aux forces qu'on leur applique. Ainsi peut-on observer que la résistance au changement dans les corps observables apparaît proportionnelle à la quantité de matière

24. Voir G. W. Leibniz, Theoria motus abstracti (I67I), in Leibniz, Mathematische Schriften, VI, p. 6I-80; François Duchesneau, La Dynamique de Leibniz, Paris, Vrin, I994, p. $15-67$.

25. Béguelin, Sur deux propriétés, p. 34I. 
qui les constitue. Mais cet effet doit être compris comme l'expression sommative de degrés de rénitence associés aux moindres systèmes d'éléments dont les corps sont constitués. La formule est ainsi résumée par Béguelin:

Il est [...] très probable que le système le plus simple, le moins compliqué, des premiers éléments de la matiere a un degré fini $\&$ déterminé de résistance au changement d'état, quoique ce degré, incomparablement plus petit que le moindre de ceux que nos sens peuvent éprouver, doive être absolument insensible pour nous. Mais le plus petit corpuscule de matiere que nous puissions apercevoir, doit déjà être un aggrégé d'un nombre prodigieux de systèmes élémentaires subordonnés \& compliqués entr'eux; la somme des inerties propres à chacun d'eux constitue par conséquent l'inertie de ce corpuscule, comme la somme des inerties de plusieurs corpuscules constitue la renitence totale du corps plus volumineux qu'ils composent ${ }^{26}$.

Ce modèle a le mérite de cumuler les rapports d'adéquation caractéristiques des hypothèses bien fondées: simplicité, continuité analogique par rapport aux données d'expérience, accord avec les principes fondamentaux et les lois de la dynamique, extension du pouvoir d'expliquer les phénomènes. C'est un édifice de concepts bien articulés, mais, par -delà ces traits méthodologiques, y adhérer représente une affaire de choix et de conviction intime à l'instar de ce qui vaudrait pour d'autres théories métaphysiques, plus ou moins analogues.

\section{Quel fondement pour l'espace?}

Le dernier mémoire que je prendrai en compte visait la Conciliation des idées de Newton et de Leibnitz sur l'espace et le vuide: il fut lu à la séance de l'Académie du I 2 octobre $1769^{27}$. Il est construit sur la base d'une réinterprétation des concepts antinomiques avancés par les deux auteurs, suivant le clivage entre le point de vue géométrique et physique d'une part, et le point de vue métaphysique de l'autre ${ }^{28}$. L'espace absolu selon Newton y est présenté comme un mode abstrait de représentation des corps, mode dont la réalité tient au seul fait que les propriétés correspondantes se trouvent actualisées dans les entités du monde physique. De façon parallèle, la thèse leibnizienne relative à l'espace est présentée comme si l'espace correspondait à une relation, celle "des êtres qui existent en même temps ${ }^{29}$ ». Et, en tant que telle, cette relation apparaît entièrement compatible avec la "notion physique et mathématique de l'espace pur ou du vuide ${ }^{30}$ », car l'ordre des êtres qui coexistent peut de fait se réaliser dans un système physique où les

26. Ibid., p. 342-343.

27. Nicolas Béguelin, «Conciliation des idées de Newton et de Leibnitz sur l'espace et le vuide ", Histoire, I77I, p. 344-360.

28. Voir ibid., p. 346.

29. Ibid., p. 356 .

30. Ibid., p. 356 . 
corps et leurs éléments sont disposés à distance les uns des autres, sans que d'autres éléments matériels ne leur soient nécessairement interposés de façon à assurer une juxtaposition sans faille.

La divergence entre les philosophes ne porte pas sur la possibilité, et donc sur le caractère non contradictoire de la notion d'espace pur ou absolu, mais sur l'existence effective de cet espace, et donc sur celle du vide, dans l'univers physique, existence que professe Newton et que conteste Leibniz. Or Leibniz soutenait ici la négative pour des raisons métaphysiques, liées aux "premiers principes et [à] la liaison des choses $^{31}$ ", alors que l'argumentation newtonienne se fondait sur des considérations que l'on pouvait ultimement tenir pour justifiées par analogie avec les données de l'expérience ${ }^{32}$.

Béguelin procède donc sur cette base à l'analyse des arguments physiques évoqués par les tenants des deux positions scientifiques antagonistes. Il ne récuse certes pas la possibilité de concevoir l'interaction des corps en mouvement dans un milieu plein, mais cette interaction n'est possible qu'en admettant des fluides subtils, sources d'impulsion et pénétrant les pores des corps, sans que leurs parties soient dotées de pesanteur assignable: la composition particulaire de ces fluides subtils et en quelque sorte impondérables requerrait d'ailleurs que la raison suffisante en fût relancée en termes de fluides encore plus subtils et plus impondérables, mais dont les éléments fussent néanmoins extrêmement denses, et cela en une régression sans terme. À l'encontre de cette hypothèse complexe, le modèle newtonien des globes ou corpuscules espacés présente l'avantage d'une relative simplicité et d'une intelligibilité ou vraisemblance plus manifeste. Ce sont d'ailleurs ces caractères gnoséologiques de l'hypothèse que Béguelin retient essentiellement en sa faveur:

Rien n'empêche donc de concevoir que la matiere est distribuée \& placée actuellement par globes isolés, comme les premieres loix de la nature semblent l'exiger; \& l'on n'a pas à craindre que l'intervalle vuide qui seroit entre ces mondes ne facilitât leur rapprochement, ou la chûte des uns sur les autres, puisque le mouvement rectiligne qui leur a été imprimé par le Créateur tendroit plutôt à les séparer de plus en plus, si la force de la pesanteur ne les conservoit dans leur distance actuelle ${ }^{33}$.

31. Ibid., p. 348 .

32. C'est ce que Newton soutenait dans le scholie suivant les définitions initiales dans les Philosophice naturalis principia mathematica. Voir Isaac Newton, Mathematical Principles of Natural Philosophy, F. Cajori (dir.), Berkeley, University of California Press, I962, I, p. 6: «I do not define time, space, place, and motion, as being well known to all. Only I must observe, that the common people conceive those quantities under no other notions but from the relation they bear to sensible objects ». De là suit la caractérisation du temps absolu, de l'espace absolu, du lieu absolu et du mouvement absolu.

33. Beguelin, Conciliation, p. 352. 
Cette hypothèse doit néanmoins être mise en balance avec le modèle élaboré par Euler concernant la nature de la lumière et qui semble en impliquer le rejet ${ }^{34}$. La structure mathématique des modèles n'est pas en cause ici, mais seulement les concepts physiques que les hypothèses déploient respectivement. Soit la lumière est constituée par une émission réelle de corpuscules lumineux, soit elle se réduit à l'effet de la pression exercée par le corps lumineux sur l'éther en tant que milieu fluide des cieux. Euler soutenait que le modèle de l'émission corpusculaire enveloppait deux implications problématiques. En étant source d'émission, le corps céleste lumineux tendrait à une déperdition importante de matière et donc de masse. Et, par ailleurs, les corpuscules émis tendraient à remplir intégralement l'espace interstellaire présumé initialement vide. S'appuyant sur divers travaux scientifiques, dont ceux de Patrice d'Arcy sur la rémanence des impressions lumineuses ${ }^{35}$, Béguelin tente de moduler l'hypothèse de l'émission en vue de l'immuniser contre l'objection d'Euler. Il imagine des mouvements alternés de pulsation affectant la source émettrice: d'où la discontinuité des émissions. Par ailleurs, l'effet imprimé par la lumière sur la rétine, s'il est présumé ponctuel dans sa production, peut s'avérer temporairement rémanent dans la perception qu'il engendre, comme les expériences réalisées par d'Arcy semblent l'établir. Compte tenu d'un espacement suffisant des particules successivement émises et de la vitesse de la lumière, on peut légitimement transposer le modèle newtonien comme s'il impliquait des distances vides immenses entre les corpuscules infimes successivement impliqués dans la transmission du rayonnement depuis sa source sidérale. En vertu de la discontinuité et de l'espacement des émissions, on peut aussi se représenter que la source lumineuse ne subirait qu'une atténuation insignifiante de sa masse mesurée selon la durée la plus longue des observations humaines. Ces hypothèses accessoires permettent ainsi à Béguelin d'accréditer une théorie newtonienne révisée de la lumière et, avec elle, la présupposition d'un vide physique. Ses recherches visant, dans la foulée de cette analyse, la réalisation d'expériences cruciales sur la nature de la lumière lui apporteront une certaine notoriété dans les milieux scientifiques ${ }^{36}$.

Or, suivant l'approche conciliatrice qu'il adopte, s'agissant des fondements de la philosophie naturelle, Béguelin doit justifier la compatibilité d'un tel modèle avec les raisons d'ordre métaphysique auxquelles il tend à réduire la conception leibnizienne de l'espace. Ces raisons tiennent à l'ordre réciproque des corps les uns par rapport aux autres, dont on peut supposer

34. Voir Leonhard Euler, "Nova theoria lucis et colorum», Opuscula varii argumenti, Berlin, Haude et Spener, I746-I75 I, I, p. I-45.

35. Voir Chevalier Patrice d'Arcy, "Mémoire sur la durée de la sensation de la vue ", Histoire, I768, p. 439-45I.

36. Voir à ce sujet Casparus Hakfoort, «Nicolas Béguelin and his Search for a Crucial Experiment on the Nature of Light", Annals of Science, 39 (I982), p. 297-3 IO. 
qu'il persiste identique à lui-même, que les corps soient séparés par des portions de vide ou qu'ils le soient par des fluides subtils qui seraient intercalés entre eux. Il est certes possible de souligner que cette équivalence des hypothèses ne serait pas recevable d'un point de vue leibnizien, puisque les raisons suffisantes du lieu des corps feraient défaut si les interstices entre eux étaient réellement vides. Mais cette considération ne retient pas l'attention de Béguelin, puisque, du point de vue physique, l'hypothèse d'un fluide subtil n'ajouterait qu'un réquisit inutile, et donc superfétatoire, à l'explication des phénomènes dans leur ordre.

Est-ce à dire toutefois que l'ordre de coexistence des corps ne serait qu'une implication découlant de l'existence d'un espace pur ou absolu? Confronté à cette supposition dérivée de la philosophie naturelle de Newton, Béguelin se dissocie de ce qu'elle implique en matière de priorité ontologique. Il pose que l'ordre de coexistence des corps résulte de l'arrangement idéal des essences que l'entendement divin conçoit au fondement du meilleur des mondes possibles. Telle est la racine ontologique de la notion d'un espace abstrait et uniforme que l'on ne saurait tenir pour absolu au sens newtonien: le fondement en serait en effet l'ordre des essences dont les corps, par leur coexistence, constitueraient l'expression contingente.

Pour réaliser la jonction de cette thèse métaphysique d'inspiration leibnizienne avec la reconnaissance d'un ordre physique possible, voire même vraisemblable, dont le vide serait partie intégrante, Béguelin interprète l'impossibilité du vide dans la nature selon Leibniz comme signifiant la plus grande perfection et donc la plus grande liaison possible des substances selon le plan de la création. Dans l'ordre des phénomènes, cette plus grande liaison possible se transpose en l'arrangement organique des corps, celui-ci pouvant, voire devant s'accomplir par une certaine implication de vide physique dans l'interrelation des corps, en vertu d'un réquisit d'harmonie. «M. Leibniz, soutient Béguelin, pouvoit si peu nier l'existence du vuide physique que ce vuide est une suite naturelle de son système sur la combinaison des éléments simples ${ }^{37}$.» Or que faut-il entendre ici par "suite naturelle» ? Il ne saurait s'agir d'une pure et simple déduction de l'ordre physique à partir de l'ordre métaphysique, mais d'une forme de compatibilité entre des représentations qui ont leur source dans des modes distincts de connaissance, selon que ces représentations se justifient respectivement par des arguments tenus pour a priori ou a posteriori. Il s'agit là ultimement de l'ajustement analogique de deux discours présumés rationnels qui se distinguent par leurs objets et par leurs techniques de preuve. Il ne fait pas de doute que Béguelin a eu le mérite de tenter de les harmoniser, c'est-à-dire de les insérer en un même système intégré de concepts interprétatifs qui pût assurer la liaison entre des notions apparemment incompatibles. C'est ce que traduit bien cette déclaration synthétique au sujet du vide: 
Mais que dirons-nous du sentiment de ces grands hommes sur l'existence du vuide? Si Newton a prouvé qu'il n'est pas possible que les mouvemens s'exécutent \& se conservent dans le plein absolu, Leibniz n'a pas moins prouvé que la nature n'admet point de vuide, \& que son idée répugne aux loix éternelles de la convenance. En adoptant les deux preuves, il faut nécessairement supposer une distinction entre le vuide physique \& le vide métaphysique; car deux vérités ne sauroient être en contradiction. Et pourquoi ne pourroit-on pas faire cette distinction? Elle découle de la nature \& de l'objet des deux sciences que je viens de nommer. Qu'est-ce que le vuide en Physique ? Ce n'est autre chose que l'espace pur, dont la notion, comme nous l'avons vû, n'a rien que de bien possible, $\&$ de très compatible avec la structure du monde matériel. Qu'est-ce, au contraire, que le vuide métaphysique? C'est une lacune, un défaut, une imperfection dans un tout; c'est l'absence d'une piece qui pouvoit $\&$ qui devoit entrer dans la construction de la machine; $\&$ c'est là le vuide que Leibnitz n'avoit garde d'admettre ${ }^{38}$.

\section{Conclusion}

Que conclure? Dans les mémoires qui ont retenu mon attention, Béguelin entendait montrer que les thèses leibniziennes relatives au fondement de l'ordre naturel pouvaient servir à parfaire l'architecture de théories physiques, reposant par ailleurs sur la généralisation de données d'expérience. À cette fin, il se livrait à une interprétation sélective des principes de la métaphysique de Leibniz. Y prédominaient la distinction des vérités contingentes et des vérités nécessaires, et la subordination des lois de la nature comme vérités contingentes au principe de raison suffisante et aux autres principes architectoniques. D’où le rattachement des généralisations empiriques de la philosophie naturelle à un ordre harmonique de convenance et de finalité prévalant dans l'univers. Cela valait pour la «déduction" des lois fondamentales de la mécanique que l'on ne saurait, en dépit de d'Alembert, réduire à des propositions abstraites relevant de la seule nécessité géométrique, mais que l'on devrait plutôt rapporter aux réquisits de l'harmonie universelle. Cela valait pour l'interaction des corps qui dépendait de l'attraction selon Newton et que l'on pourrait, voire que l'on devrait rattacher aux principes de l'interrelation harmonique entre monades, éléments ultimes de l'ordre naturel et fondements de la liaison, de la composition et de l'interaction des corps. Béguelin, dans cette recherche de fondement ontologique pour l'ordre des phénomènes, n’hésite pas à concevoir des hypothèses aptes à représenter le système d'intégration de forces mouvantes élémentaires, système qui soustendrait l'inertie des corps selon la mécanique newtonienne. Et il se livre à une démarche similaire pour concilier la physique du vide et la conception géométrique de l'espace absolu avec une métaphysique leibnizienne qui fait de l'espace un ordre de coexistence dépendant de l'harmonie des éléments monadiques et exprimant le plan intelligible du monde suivant l'ordre de 
composition des réalités naturelles mêmes. De telles synthèses ne seraient ultimement possibles qu'en réduisant la philosophie naturelle des Newtoniens aux seules inférences inductives et analogiques d'une science limitée aux corrélations empiriques, et elles ne seraient corrélativement possibles qu'en assimilant les principes de la philosophie naturelle leibnizienne à des constructions métaphysiques qui serviraient à établir un système rationnel possible des lois de la nature. Sans doute pourrait-on estimer qu'il ne s'agirait là que d'un habile bricolage spéculatif. Je préfère pour ma part y voir l'expression d'une démarche de fondement théorique pour une philosophie de la nature qui restait à unifier dans ses modèles et ses méthodes, par voie d'hypothèses analogiquement fondées. Et l'on ne saurait professer que cette problématique ait été dénuée de pertinence. 